\title{
GTO 阻断状态的新型识别法*
}

\author{
张立吴爱国 李云德 王 江 \\ (天津大学自动化系, 天津300072)
}

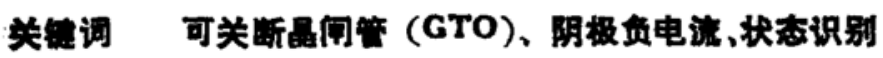

\section{1 门极一阴极结的反向霓朋击穿}

图 1 所示为 GTO 关断时的门极电路各参数的波形图, 图中 $E_{\text {off }}$ 为门极负电源电压, $G_{G}$ 为门极回路的布线电感.

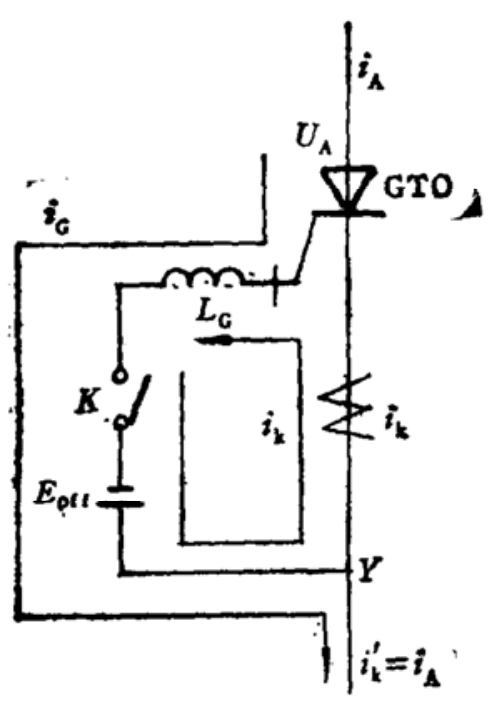

(a)

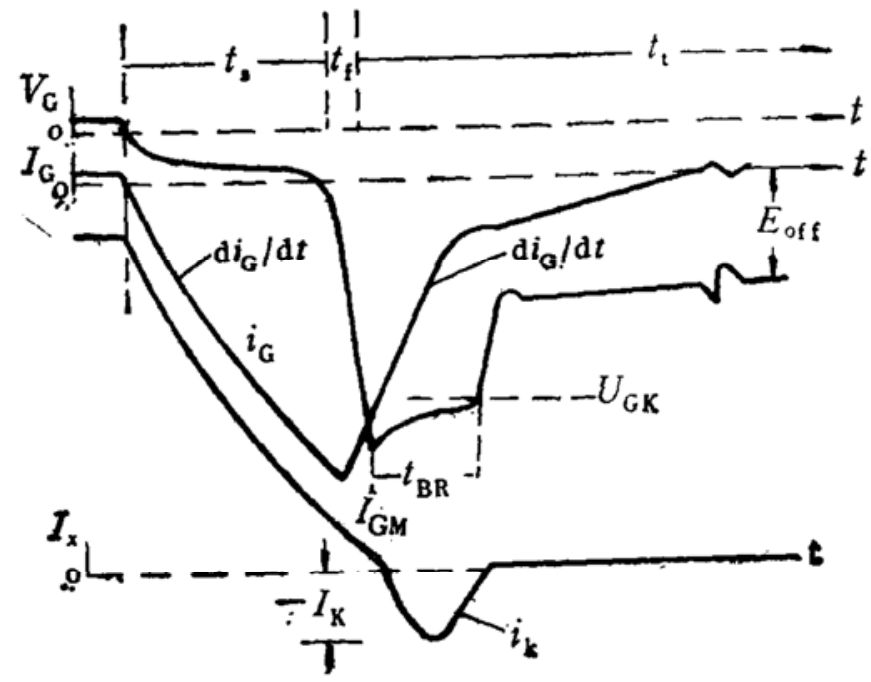

(b)

图 1 门极关断电路及各参数波形图

当开关 $\mathrm{K}$ 闭合之后, 已开通的 GTO 即进人关断过程, 由于门极负电压的作用, 从 GTO 阳极中抽出门极负电流 $i_{G}$, 其电流流向如图 1 (a) 所示, 此时阴极实际流过的电流为正向电 流 $i_{k}^{\prime}$. 由于布线电感 $L_{\mathrm{G}}$ 的存在, 在门极负电流 $i_{\mathrm{G}}$ 达到最大值 $I_{\mathrm{GM}}$ 之前, 电感 $L_{\mathrm{G}}$ 阻碍门 极负电流的增加, 在 $I_{\mathrm{GM}}$ 之后, 它又阻碍门极负电流的下降, 也就是说, 在贮存时间内电感 $L_{\mathrm{G}}$ 览能, 而在下降时间内电感 $L_{\mathrm{G}}$ 放能. GTO 关断过程中门极负电流 $i_{\mathrm{G}}$ 和门极-阴极之间的 电压以及阴极电流的变化过程如图 1(b) 所示.

在电感 $L_{\mathrm{G}}$ 放能的过程中, 电感 $L_{\mathrm{G}}$ 上产生的电压 $\left(-L_{\mathrm{G}} \frac{\mathrm{d} i_{\mathrm{G}}}{\mathrm{d} t}\right)$ 与电源负电压 $E_{\mathrm{off}}$ 选 
加在一起作用在 GTO 门极-阴极结上. 此时门极回路中各参数的关系为:

$$
L_{\mathrm{G}} \frac{\mathrm{d} i_{\mathrm{G}}}{\mathrm{d} t}+E_{\mathrm{off}}>U_{\mathrm{GR}},
$$

式中 $U_{\mathrm{GR}}$ 为 GTO 门极-阴极的反向酎压,一般为 $16-25 \mathrm{~V}$.

若电感上的电压 $L_{\mathrm{G}} \frac{\mathrm{d} i_{\mathrm{G}}}{\mathrm{d} t}$ 与 $E_{\mathrm{off}}$ 之和大于 $U_{\mathrm{GX}}$, 门极一阴极结则会产生反向雪崩现象. 产生雪崩击穿时, GTO 阳极电流已进人下降时间的末尾, 其电流值接近于尾部电流, 此时阴 极必然产生负电流 $i_{k} . i_{k}$ 的流向由图 1(a) 给出.

尽管在设置门极电路时, 尽量缩短配线, 布线时又加以工艺考究, 但是总会存在一定布线 电感 $L_{\mathrm{G}}$. 因为 GTO 关断时, 门极负电流的下降速度 $-\frac{\mathrm{d} i_{\mathrm{g}}}{\mathrm{d} t}$ 很快, 一般可达 $60 \mathrm{~A} / \mu \mathrm{s}$ 以 上, 因而布线电感 $L_{\mathrm{G}}$ 必然产生足够高的电压, 它与 $E_{\text {off }}$ 迭加足以使门极一阴极结反向雪崩 古穿. 只要产生反向雪崩击穿, 必然产生阴极负电流 $i_{k}$. 图 2. 所示为 600A/1600V GTO 关 断阳极电流为 $500 \mathrm{~A}$ 时阴极负电流 $i_{k}$ 和门极-阴极结反向电压的波形. 由图可知, 阴极负电 流最大值为 $75 \mathrm{~A}$, 持续时间约为 $980 \mathrm{~ns}$.

实验证明, 在门极电路各参数不变的条件下, 随着可关断阳极电流的增加, 阴极负电流的 幅度和持续时间增加; 在关断相同的阳极电流时, 若门极电感增加, 阴极负电流的持续时间增 加. 由于门极电感 $L_{\mathrm{G}}$ 的增加, 使门极负电流的下降速度减小, 所以 $L_{\mathrm{G}}$ 增加时阴极负电流 有下降的趋势。

阴极负电流必然使门极一阴极结的损耗增加, 但是只要雪崩时间不长, 这种损坏不会超过 门极一阴极结的允许功耗范围 ${ }^{[1]}$.

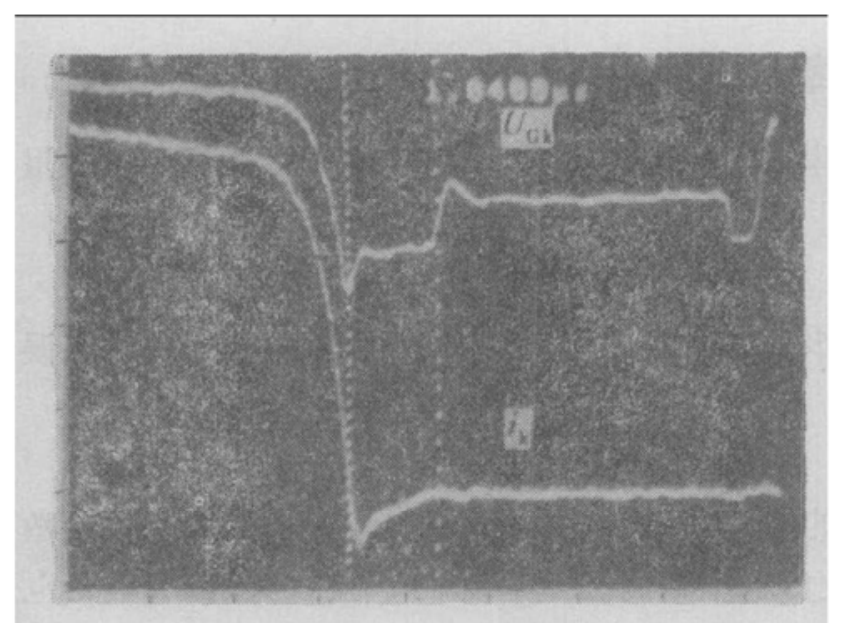

图2 阴极负电流与门极一阴极结反向电压波形 i: $1 \mu \mathrm{s} / \mathrm{div}, U_{\mathrm{GK}}: 10 \mathrm{~V} / \mathrm{div}, i_{k}: 100 \mathrm{~A} / \mathrm{div}$

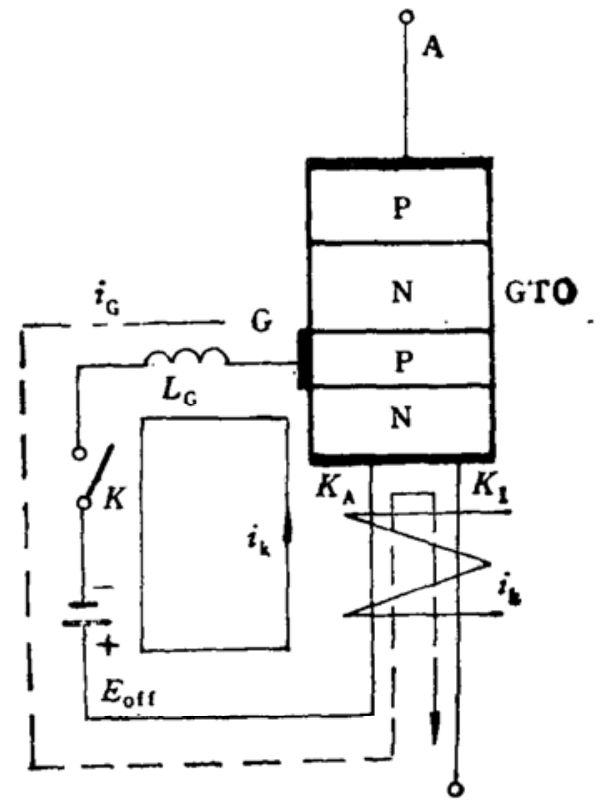

图3阴极负电流直接检测法

\section{2 阴极负电流的检测}

为了使用方便, 大容量 GTO 有主阴极引线端 $K_{1}$ 和辅助阴极引线端 $K_{A}$. 直接从 $K_{A}$ 
端检测门极一阴极结雪崩击穿时的阴极负电流难以实现，检测阴极负电流的方法可有如下两 种:

\section{1 直接检测法}

因为 GTO 辅助阴极引线端 $K_{\mathrm{A}}$ 的存在,要想检测门极-阴极结的反向雪崩电流, 必须按 照图 3 所示办法连接电流传感器. 当门极-阴极产生雪崩击穿现象时,阳极尾部电流继续从门 极抽出形成门极负电流. 因为此电流从电流传感器中流人又流出, 所以在传感器的输出中不 反映此电流。另外,雪崩击穿电流只流入电

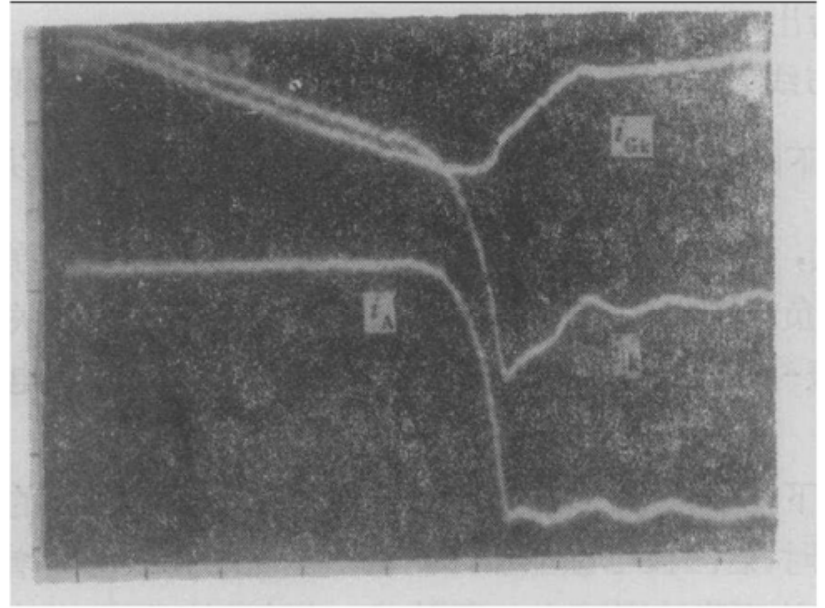

图 4 用间接法测出的阴极负电流波形 t: $1 \mu \mathrm{s} / \mathrm{div}, i_{A}: 100 \mathrm{~A} / \mathrm{div}, i_{k}: 50 \mathrm{~A} / \mathrm{div}$ 流传感器，因而可从电流传感器中直接检测 出阴极正负电流. 图 3 中阴极电流 $i_{k}$ 所示 为雪崩击穿电流，此时主阴极电流已下降为 零.

\section{2 间接检副法}

用阳极电流 $i_{\mathrm{A}}$ 与关断时门极负电流 $i_{\mathrm{A}}$ 的迭加可以求出阴极负电流 $i_{k}$ 的极性和大 小.

从图 1(a) 可以看出, 在节点 $\mathrm{Y}$ 有:

$$
\begin{array}{cc} 
& i_{k}^{\prime}=i_{k}+i_{\mathrm{G}}, \\
\because & i_{k}^{\prime}=i_{\mathrm{A}}, \\
\therefore & i_{\mathrm{A}}=i_{k}+i_{\mathrm{G}}, \text { 即 } i_{k}=i_{\mathrm{A}}-i_{\mathrm{G}} .
\end{array}
$$

由此可知:

门极-阴极雪崩前, $i_{k}>0$;

门极一阴极雪崩后, $i_{k}<0$.

图 4 所示为 $600 \mathrm{~A} / 1300 \mathrm{~V}$ GTO 用间接法检测出的阴极负电流的波形, 与直接检测法相 比两者结果相同.

\section{GTO 阻断状态的识别}

在 GTO 逆变器的互锁控制、故障诊断和事故记录中, 很有必要可靠地识别 GTO 的阻 断状态. 根据 GTO 关断时各参数的变化识别 GTO 阻断状态的办法通常有如下两种:

\section{1 直接监测阳报电流或阳极电压法 ${ }^{[2]}$}

这种方法曾用于晶闸管电路, 其缺点是阳极或阴极结断开时, 可把 GTO 的损坏状态误 识为正常阻断状态.

\section{2 门极电流和电压监测法 ${ }^{[3]}$}

由图 1(b) 可知, 在贮存时间末尾, GTO 的中心结已开始阻断. 识别这一点的有关参数 可确认 GTO 的阻断状态. 通常联合监测门极负电压和门极负电流, 或者联合检测门极负电 压与门极负电流减小时的电流变化率来确定 GTO 的阻断状态. 这些方法需要在门极电路 中串人电流传感器或微分变压器,易引人干扰信号, 影响检测信号的准确性.

本文提出一种新型的 GTO 阻断状态识别法. 从图 1(b) 所示阴极电流的波形中可知, 在门极-阴极结雪崩击穿之后阴极电流转为负值. 因为阴极负电流出现之时, 不但 GTO 中 心结已经恢复阻断, 而且门极-阴极结也已恢复阻断, 所以识别阴极负电流可以更可靠地确认 
GTO 的阻断状态. 在这种识别方法中信号电流大不易引入干扰噪音, 测试方便, 便于推广应 用。

\section{4 结 论}

由于大容量 GTO 门极电路布线电感的作用, 在下降时间末尾, 门极一阴极结必然产生幅 度相当大的阴极负电流. 识别这一负电流即可识别 GTO 的阻断状态. 这种状态识别可在 GTO 逆变电路的互锁控制、故障诊断和事故记录等领域中应用.

致谢 本项研究得到燕山大学自动化系张纯江、刘彦民和渿汉宏等先生的热情帮助, 特此 致谢.

\section{考文嗝}

[1] Ho, Y. Y., IEEE Transactions on Industrial Electronics, Vol. IE-33, No. 3, August 1986.

[2] Seki, N. et al., PESC'83, 1983, 183-193.

[3] Salzmann, T. et al., in Conf. Rec. of 1986 IEEE Industry Application Sociezy Annual Meeting, $458-463$. 